\title{
Special issue on artificial immune systems
}

\author{
Jon Timmis · Paul Andrews · Emma Hart
}

Received: 14 September 2010 / Accepted: 16 September 2010 / Published online: 14 October 2010

(C) Springer Science + Business Media, LLC 2010

The field of artificial immune systems (AIS) is a diverse area of research that bridges the disciplines of immunology and engineering. AIS algorithms are typically developed from the abstraction of immune system theories, processes and agents, and they have been applied to a wide variety of engineering applications including computer security, fault tolerance, data mining and optimisation. More recently there has been a growing trend within AIS to facilitate closer interaction between the domains of immunology and engineering through the use of various mathematical and computational modelling approaches. These have included dynamical systems analysis, agent-based modelling and cellular automata. The resulting models serve a dual purpose: to improve understanding of the biological domain, and to aid the development of more biologically inspired AIS for engineering problems.

The field of swarm intelligence (SI) encompasses a wide range of scientific and engineering disciplines to explore and exploit the complex behaviours that arise from groupings of agents such as social insects or animals. Research in this field incorporates many decentralised and distributed systems that exploit the collective behaviour that emerges from the interaction of individual agents with each other and their environment. This perspective affords a natural link between SI and AIS: many immune algorithms operate in a very similar manner with populations of immune agents exhibiting similar high-level collective behaviours; it has furthermore been suggested by several authors that the natural immune

\footnotetext{
J. Timmis $(\bowtie)$

Department of Computer Science and Department of Electronics, University of York, Helsington, York, UK

e-mail: jtimmis@cs.york.ac.uk

P. Andrews

Department of Computer Science, University of York, Helsington, York, UK

e-mail: psa@cs.york.ac.uk

E. Hart

School of Computing, Edinburgh Napier University, Merchiston Campus, Edinburgh, UK

e-mail: e.hart@napier.ac.uk
} 
system itself should be considered as swarm-like. This special issue of the Swarm Intelligence journal therefore presents state-of-the-art results in the area of AIS to an SI audience, showcasing novel results from both an engineering and immunological perspective.

The special issue solicited high quality articles that exploited immune-inspired mechanisms to address problems in engineering or developed models to aid understanding in immunology. Following a rigorous peer review process, four papers are published in this issue, which cover the broad scope of AIS research. The first paper "On artificial immune systems and swarm intelligence" by Timmis et al. considers the relationship between the fields of SI and AIS. The paper draws parallels between the two fields from both a science and engineering perspective, but it also highlights some differences. Rather than seeking to place the fields on a competitive footing, the paper draws attention to their complementarities offering opportunities for researchers in SI and AIS to work together to effectively solve complex engineering problems.

"Words, antibodies and their interactions" by Nanas et al. addresses the engineering problem of building a user profile for adaptive information filtering. The paper exploits an immunological model of antibody-antibody interactions which provides possible explanations for self-tolerance and increased diversity in the immune repertoire in order to model correlations between words in text documents. The resulting system is shown to be able to both assess the relevance of documents to a user's interests and furthermore, to adapt to changes in them.

The article "Scale invariance of immune system response rates and times: perspectives on immune system architecture and implications for artificial immune systems" by Banerjee and Moses models the phenomenon whereby immune response rates are independent of body size. Both ordinary differential equations and agent-based models are used in the investigation, which suggest that immune modularity balances the trade-off between pathogen detection and immune response. The authors conclude by examining the implications of immune modularity in an engineering context, namely an immune-inspired distributed robot controller.

Sorathiya et al. examine how modelling techniques can be used to help assess the effects of drug therapies in "An integrated modelling approach for R5-X4 mutation and HAART therapy assessment". A population-based stochastic model of R5-X4 phenotype-switching in HIV is described that incorporates the dynamics of the HAART drug therapy, and it shows how the drug therapy can slow the progress of the disease. The model is then used to examine the benefits of integrating formal model checking and mathematical models for drug therapy validation.

We would like to thank Marco Dorigo, the Editor-in-Chief of Swarm Intelligence, for giving us the opportunity to construct this special issue, and for providing invaluable guidance. Finally, we hope that you, the reader of this special issue, find the articles presented within a useful resource for your own SI and AIS investigations. 